\title{
Feedback Regulation of N Fixation in Frankia-Alnus Symbiosis Through Amino Acids Profiling in Field and Greenhouse Nodules
}

\author{
Anne-Emmanuelle Hay, ${ }^{1,2,+}$ Aude Herrera-Belaroussi, ${ }^{1}$ Marjolaine Rey, ${ }^{1,2}$ Pascale Fournier, ${ }^{1}$ \\ Philippe Normand, ${ }^{1}$ and Hasna Boubakri ${ }^{1,+}$ \\ ${ }^{1}$ Université de Lyon, F-69361, Lyon, France, Université Claude Bernard Lyon 1, CNRS, UMR 5557, INRA UMR1418, Ecologie \\ Microbienne, F-69622, Villeurbanne, France \\ 2 Université Claude Bernard Lyon 1, CNRS, UMR 5557, INRA UMR1418, Ecologie Microbienne, Centre d'Etude des \\ Substances Naturelles
}

Accepted 19 November 2019.

\begin{abstract}
Symbiosis established between actinorhizal plants and Frankia spp., which are nitrogen-fixing actinobacteria, promotes nodule organogenesis, the site of metabolic exchange. The present study aimed to identify amino acid markers involved in Frankia-Alnus interactions by comparing nodules and associated roots from field and greenhouse samples. Our results revealed a high level of citrulline in all samples, followed by arginine (Arg), aspartate (Asp), glutamate (Glu), $\gamma$-amino-nbutyric acid (GABA), and alanine (Ala). Interestingly, the field metabolome approach highlighted more contrasted amino acid patterns between nodules and roots compared with greenhouse samples. Indeed, 12 amino acids had a mean relative abundance significantly different between field nodule and root samples, against only four amino acids in greenhouse samples, underlining the importance of developing "ecometabolome" approaches. In order to monitor the effects on Frankia cells (respiration and nitrogen fixation activities) of amino acid with an abundance pattern evocative of a role in symbiosis, in-vitro assays were performed by supplementing them in nitrogen-free cultures. Amino acids had three types of effects: i) those used by Frankia as nitrogen source (Glu, Gln, Asp), ii) amino acids stimulating both nitrogen fixation and respiration (e.g., Cit, GABA, Ala, valine, Asn), and iii) amino acids triggering a toxic effect (Arg, histidine). In this paper, a N-metabolic model was proposed to discuss how the host plant and bacteria modulate amino acids contents in nodules, leading to a fine regulation sustaining high bacterial nitrogen fixation.
\end{abstract}

Keywords: arginine, aspartate, bacteria-plant symbiosis, citrulline, glutamate, glutamine, metabolism, non-rhizobium nitrogen fixation

Mutualistic symbioses are striking examples of successful coevolution promoting plant growth by facilitating access to

${ }^{\dagger}$ Corresponding authors: A.-E. Hay; hay.de-bettignies@univ-lyon1.fr and H. Boubakri; hasna.boubakri@univ-lyon1.fr

*The $\boldsymbol{e}$-Xtra logo stands for "electronic extra" and indicates that a supplementary table file is published online.

The author(s) declare no conflict of interest.

๑) 2020 The American Phytopathological Society scarce nutrients and, particularly, to nitrogenous compounds. Certain plants have developed highly sophisticated systems to interact with nitrogen-fixing bacteria. These bacteria together with archaea are the unique organisms able to reduce dinitrogen into ammonium through the action of the nitrogenase complex and accessory enzymes (Dos Santos et al. 2012; Zehr et al. 2003). In exchange for nitrogenous compounds that permit colonization of N-limited ecosystems, plants provide carbon, energy, and a protected niche to diazobacteria. After a specific and complex crosstalk, these plants develop specialized organs, called nodules, that house diazobacteria. Nodulation involves two types of diazobacteria, rhizobia that associate symbiotically with legumes (Fabales) and with one nonlegume genus, Parasponia (Rosales), and filamentous actinobacteria of the genus Frankia, able to induce nodulation on a very diverse group of plants from the Fagales, Rosales, and Cucurbitales, collectively called "actinorhizal plants" (Hocher et al. 2011; Martin et al. 2017).

Nitrogen assimilation, storage, and transport processes have been studied to understand the interaction between the host plant and its bacterial symbiont. In several legumes, ammonium, the immediate product of bacterial nitrogen fixation, is either directly exported through a specific ammonium transporter $(\mathrm{AmtB})$ or through membrane diffusion to the cytosol of infected plant cells (Lodwig et al. 2003; Prell and Poole 2006). However, ammonium is highly reactive and may interfere with the $\mathrm{pH}$ homeostasis. To ensure its rapid conversion into more inert $\mathrm{N}$ compounds, ammonium is assimilated by the cytosolic glutamine (Gln) synthetase (GS) and glutamate (Glu) synthase (Gln oxoglutarate aminotransferase [GOGAT]) cycle. The resulting Glu can then be taken up by the bacterium from the plant infected cells via Aap/bra channels and can be incorporated into aspartate (Asp) and asparagine through the action of an Asp amino-transferase (AaT) (Lodwig et al. 2003; Prell et al. 2009b; Rastogi and Watson 1991). In return, the plant shuts down ammonium assimilation in rhizobia bacteroids and provides amino acids to bacteroids that are auxotrophic for them. It was suggested that this feedback provision of amino acids by the plant ensures a higher efficiency of bacterial nitrogen fixation and coupling of the two metabolisms, to modulate the transfer of carbohydrates and make it dependent on the export of ammonium (Lodwig et al. 2003; Prell and Poole 2006; Prell et al. 2009a and b).

In actinorhizal nodules, symbiotic interactions between Frankia spp. and the host plant are less understood, partly 
because of the lack of genetic tools for bacterial transformation (Kucho et al. 2009)_although transformation protocols have recently been developed, which could open perspectives (Gifford et al. 2019; Pesce et al. 2019). A fundamental difference between Frankia spp. and rhizobia lies in the ability of Frankia spp. to fix nitrogen not only inside its host plant cells during symbiosis but also in the free-living culture state. In Frankia spp., the nitrogenase involved in nitrogen fixation is located in specific structures called "diazovesicles" (except inside Casuarina and Allocasuarina nodules), which are characterized by multiple hopanoid-rich layers to protect the enzyme against oxygen entry (Berry et al. 1993). In actinorhizal plants, ammonium resulting from nitrogen fixation can be directly exported to plant cells or first converted into amino acids (e.g., Gln or Glu) via the GS/GOGAT cycle, and this N-metabolic flux seems to differ according to the plant species (Berry et al. 2011). In the case of Datisca glomerata, the whole cycle is activated in Frankia cells, whereas only Frankia GS is found modestly upregulated in Alnus glutinosa nodules (Alloisio et al. 2010; Berry et al. 2004). In A. glutinosa and D. glomerata, the cytosolic GS has also been described as active (Berry et al. 2004; Guan et al. 1996; Hirel et al. 1982), suggesting that export of either ammonium or $\mathrm{Gln} / \mathrm{Glu}$ is possible. In addition, in D. glomerata nodules, bacterial reactions involved in the arginine (Arg) pathway are also induced, suggesting that Arg (reported highly abundant in nodules) could be another amino acid involved in $\mathrm{N}$ translocation from Frankia cells to plant cells (Berry et al. 2011). This assumption is also suggested for Alnus nodules by the high abundance of citrulline (Cit) (Berry et al. 2011; Valverde and Huss-Danell 2008) involved in the Arg pathway. Other amino acids could also play an important role in $\mathrm{N}$ translocation, such as asparagine, which is preferentially stored in the nodules of genera Myrica, Hippophae, Elaeagnus, Ceanothus, and Casuarina (Wheeler and Bond 1970), and the composition of exported $\mathrm{N}$ compounds in the xylem varies depending on the actinorhizal plant (Valverde and Huss-Danell 2008).

The $\mathrm{N}$ metabolism involved in actinorhizal symbiosis still remains challenging to describe, and most studies were performed on nodules resulting from young seedlings grown under greenhouse conditions, colonized by a small set of Frankia strains (Brooks and Benson 2016; Persson et al. 2016; Tonin et al. 1990) or crushed field nodules (Baker et al. 1997; Blom et al. 1981; Leaf et al. 1958; Tonin et al. 1990; Wheeler and Bond 1970). Although nodule and root samples collected from the field could give a broader view of symbiotic interactions, they have been very rarely used for metabolomic analyses (Hay et al. 2017; Tonin et al. 1990; Virtanen and Miettinen 1952).

The purpose of the present study was to gain more insights on $\mathrm{N}$ metabolism of one actinorhizal plant model, the Alnus genus. Alnus species are widely distributed, with A. glutinosa being the most-studied species (Pozzi et al. 2015). Here, we aimed to unravel the amino acids present in A. glutinosa tissues by comparing nodules and associated root contents. Our main objective was to perform these metabolic profiling analyses combining both field and greenhouse samples in order to identify in a more exhaustive way amino acid contents involved in Frankia-Alnus interactions. Comparing both types of samples also allowed a discussion of the impact of environmental conditions when studying the eco-metabolome (i.e., metabolome described from in-natura samples) of plant-bacteria symbiotic interactions. We also combined metabolic profiling with in-vitro physiological monitoring of these $\mathrm{N}$ metabolites on the Alnus-infective Frankia alni ACN14a strain (Normand and Lalonde 1982), in order to understand the roles of amino acids in nodules. The results allowed us to discuss how the plant would control the growth and nitrogen metabolism of its symbiont through its amino acids content and to propose a model for N storage, translocation, and regulation in the AlnusFrankia symbiosis.

\section{RESULTS}

Comparison of amino acid profiles of nodule and root samples from field versus greenhouse.

A total of 20 amino acids were detected in root and nodule samples obtained from field or greenhouse experiments (Fig. 1). In all samples, Cit was the most abundant amino acid (38.7 to $60.5 \%$ of mean relative abundance within roots and nodules from greenhouse or field), followed by Arg and Asp (3.9 to 17.1\% and 4.4 to $7.4 \%$, respectively) (Fig. 1A; Supplementary Table S2). Glu, $\gamma$-amino-n-butyric acid (GABA), alanine (Ala), and valine (Val) were the next four most abundant amino acids (mean relative abundance between 1.2 and $8.3 \%$ ). All the other amino acids were present with a mean relative abundance under $3 \%$ regardless of the origin of the sample (Fig. 1B; Supplementary Table S2).

A principal component analysis (PCA) was performed on the metabolic profiles in order to compare the relative abundance of amino acids between field and greenhouse samples. Distinct metabolic profiles were observed between field and greenhouse samples for both root and nodule samples (Fig. 2). Moreover, PCA axis 1 (horizontal axis) and axis 2 (vertical axis) represented 52.3 and $16 \%$ of the total variance, respectively, with a better separation of field nodule and root samples along the first axis, suggesting a stronger contrast in amino acid contents between nodules and roots from the field compared with greenhouse samples (Fig. 2).

\section{Variations in amino acid content between nodules and associated roots.}

Amino acids specifically abundant (nodules compared with roots) were identified according to sample origin (greenhouse or field samples). We first focused on the seven amino acids with mean relative abundance in nodules and roots greater than $3 \%$ (in greenhouse, field, or both), i.e., Cit, Arg, Asp, Glu, GABA, Ala, and Val (Fig. 1A). Despite the divergence between field and greenhouse data, Arg was the only amino acid with significant differences in abundance between nodules and roots, whatever the sample origins (field and greenhouse samples). Indeed, Arg was constantly found twice more abundant in roots compared with nodules (Supplementary Table S2). For greenhouse samples, only two other amino acids were found significantly different between nodules and roots, Cit and Asp. For Cit, a 1.4 times greater content was measured in nodules compared with roots (54.4 against $38.7 \%$, respectively). In contrast, Asp was found 1.3 times and significantly more abundant in roots compared with nodules (5.7 against 4.4\%, respectively). When considering field samples, Glu and Val were significantly higher in nodule samples compared with roots with a ratio of around 1.5 to 1.8 (Supplementary Table S2). Glu was also found more abundant (although not significantly so) in greenhouse nodules compared with roots. Finally, only two amino acids (GABA, Ala) did not vary significantly between nodule and root samples, whatever the sample origins.

When we considered the 13 other amino acids with a mean relative abundance inferior to $3 \%$, only glycine was different between greenhouse roots and nodules, with a significantly greater abundance in root samples (Fig. 1B; Supplementary Table S2). Nine amino acids were different only in field samples. Indeed, histidine (His), serine (Ser), tryptophan (Trp), proline (Pro), lysine (Lys), isoleucine (Ile), phenylalanine (Phe), and tyrosine (Tyr) were significantly more abundant in nodules, whereas Asn was more abundant in roots. Three amino acids (threonine [Thr], leucine [Leu], and Gln) did not vary among samples. 
Effects of amino acids on Frankia alni ACN14a respiration and nitrogen fixation.

The effects of amino acids on Frankia respiration (iodonitrotetrazolium reduction activity [IRA]) and nitrogen fixation (acetylene reduction activity [ARA]) were tested by performing a kinetic assay with $F$. alni ACN14a as model. The seven amino acids with more than $3 \%$ mean relative abundance in either the field, greenhouse, or both were tested (Cit, Arg, Asp, Glu, GABA, Ala, and Val) as well as two other amino acids, His and Asn. These two amino acids were included in the tests because they represented strong markers of nodules or roots with a fold change between both organs greater than 2 (Fig. 1; Supplementary Table S2). Gln was also included in the experiment as a control. The effects of these 10 amino acids were tested on $F$. alni ACN14a under $\mathrm{N}$-fixing conditions (without ammonium) at two different concentrations (1 and $10 \mathrm{mM})$; three groups of amino acids were distinguishable according to their effects on $F$. alni ACN14a respiration and nitrogen fixation.

First, two amino acids (Glu and Asp) showed similar effects to those observed for the Gln control. More precisely, Gln significantly reduced nitrogen fixation activity at $1 \mathrm{mM}$ and totally inhibited it at $10 \mathrm{mM}$, as compared with the control with no amino acid supplementation (N-free) (Fig. 3A). In parallel, this amino acid significantly stimulated bacterial respiration (Fig. 3B). Glu and Asp also partially reduced Frankia nitrogen fixation, and more drastically so, at $10 \mathrm{mM}$ without causing a total inhibition, such as with Gln. Both Glu and Asp significantly stimulated bacterial respiration and growth, particularly at $10 \mathrm{mM}$. For Gln, this stimulation occurred very early in the timecourse (after 3 days of growth).
The second group of amino acids consisted in Arg and His, which inhibited both nitrogen fixation and bacterial respiration (Fig. 3C and D). These negative effects were more precisely observed for His at both concentrations tested, whereas only Arg at $10 \mathrm{mM}$ drastically inhibited the two metabolic activities. At $1 \mathrm{mM}$, Arg was comparable to the $\mathrm{N}$-free control.

The last group included amino acids resulting in a moderate or high nitrogen fixation with no or slightly positive effect on bacterial respiration and growth, as compared with the N-fixing control. Five amino acids (Cit, Val, Asn, Ala, and GABA) (Fig. 4) induced a significantly higher nitrogen fixation at both concentrations, with the highest stimulation observed for Ala $10 \mathrm{mM}$ at 7 days. In addition, we noticed a dose effect for Ala, with greater stimulation observed for a higher concentration. GABA also increased Frankia ACN14a nitrogen fixation at $10 \mathrm{mM}$ but delayed its optimum timepoint relative to the control. Indeed, after 10 days of cultivation, nitrogen fixation stopped for almost all amino acids tested except GABA. Focusing on bacterial respiration, only slight positive effects were observed with Asn (very early in the kinetics) and with Val at $10 \mathrm{mM}$ (Fig. 4B). A moderate or no effect was detected for Cit, Ala, and GABA at $1 \mathrm{mM}$ when only Cit and Ala at $10 \mathrm{mM}$ triggered a small positive effect on bacterial respiration.

\section{DISCUSSION}

Relevance of combining field-

and greenhouse-based approaches.

In order to understand $\mathrm{N}$ storage and $\mathrm{N}$ translocation in the Frankia-A. glutinosa symbiotic model, amino acid content was assessed in both field and greenhouse nodules and associated
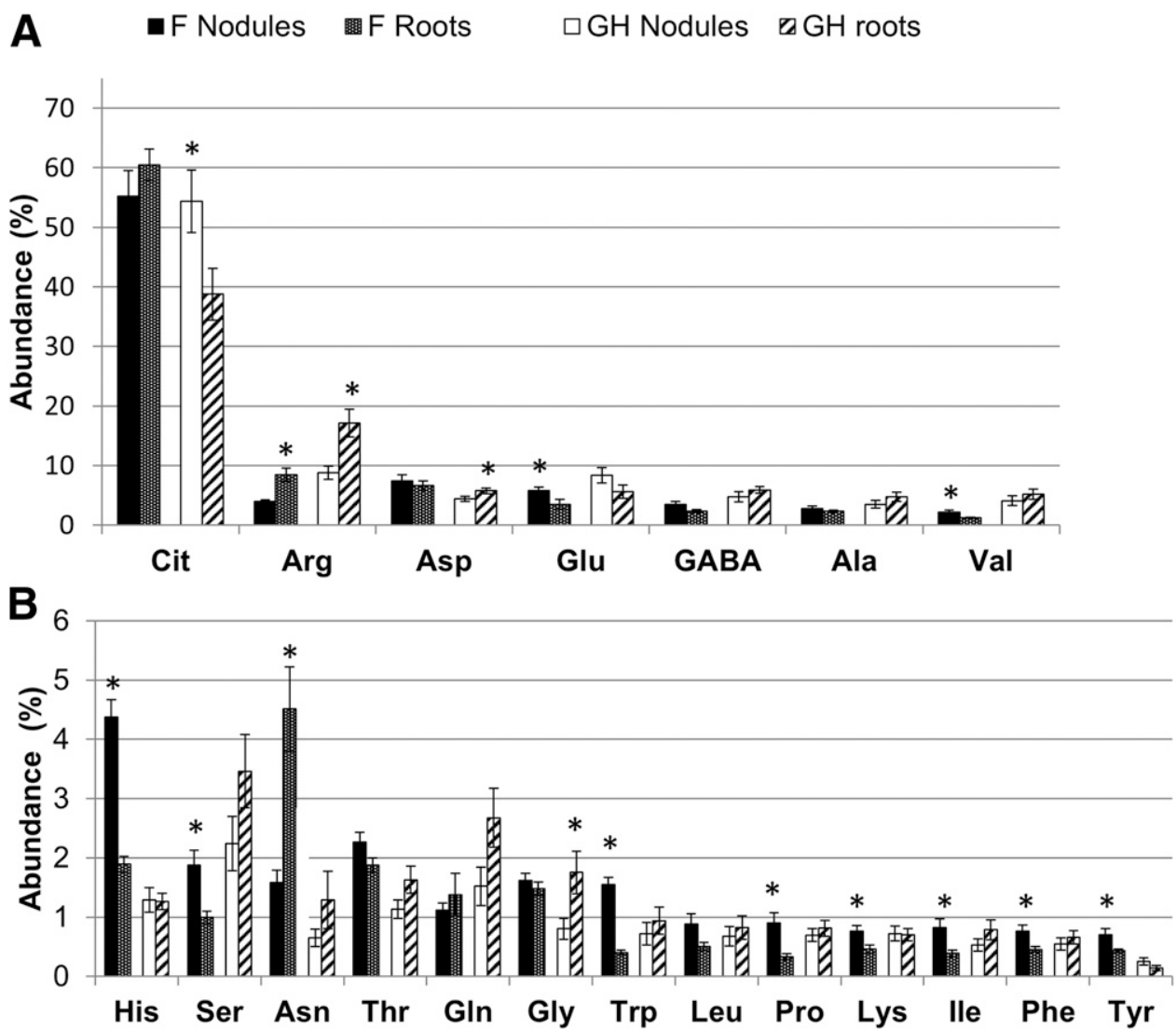

Fig. 1. A and B, Mean relative abundance of amino acids in nodules and roots for each type of sample: field (F) or greenhouse (GH). The mean relative abundance of each amino acid in all samples ( $\mathrm{F}$ and $\mathrm{GH}$ ) and organs (nodules and roots) was calculated for each amino acid in order to identify those presenting a percentage superior (A) or inferior (B) to $3 \%$. Asterisks $(*)$ indicate significant differences observed between amino acid mean relative abundance within the organ (nodule or root) from the same origin $(\mathrm{F}$ or $\mathrm{GH})(P$ value $<0.05)$. 
roots. Presently, amino acid profiles are mainly made for A. glutinosa plants grown under greenhouse conditions from several weeks to two years (Baker et al. 1997; Blom et al. 1981; Brooks and Benson 2016; Gardner and Leaf 1960; Leaf et al. 1958; Persson et al. 2016; Schubert and Coker 1981; Tonin et al. 1990; Wheeler and Bond 1970). Here, we analyzed samples from both greenhouse and field conditions. Interestingly, global metabolic profiles of amino acid contents were contrasted between field and greenhouse samples, with a stronger contrast in amino acid contents between nodules and roots from the field; 13 amino acids had a mean relative abundance significantly different between both types of organs, against only four amino acids in greenhouse samples: Arg (the only one highlighted from both field and greenhouse samples), Cit, Asp, and glycine (Gly) (significantly different only in greenhouse samples), Glu, Val, His, Ser, Trp, Pro, Lys, Ile, Phe, Tyr, and Asn (significantly different only in field samples).

These differences between field and greenhouse samples could be explained by numerous factors, such as environmental conditions or the age of host plants.

A previous study also based on greenhouse nodules of A. glutinosa inoculated with $F$. alni ACN14a showed Cit and Glu as the most abundant amino acids (Persson et al. 2016), as we observed from our greenhouse nodule samples. Other works based on plants inoculated with a different Frankia strain (CpI1) reported GABA as the most abundant amino acid in nodules, followed by Cit (16.8\%) and Glu (15\%) (Brooks and Benson 2016), suggesting that, in addition to host plant parameters, Frankia strains could also strongly influence the symbiotic metabolome. Finally, field nodules are exposed to soil microbial communities and we cannot exclude the presence of non-Frankia bacteria inhabiting nodules and, thus, their potential effects on the symbiotic metabolome. For instance, strains related to genus Micromonospora or other actinobacteria were previously isolated from field nodules (Carro et al. 2013; Ghodhbane-Gtari et al. 2010). However, their extremely low abundances in nodules (they could not be found by high throughput 16S rRNA) (Schwob et al. 2017, 2018) suggest a very low influence on nodule metabolism.

Thus, our results suggest that i) plant metabolic profiles are deeply dependent on environmental conditions, including host plant traits and Frankia strain genotype, and consequently, ii) using laboratory (e.g., greenhouse) models based on a unique set of plants and Frankia strains can give a partial view of symbiotic interactions. Here, we illustrated the relevance of performing eco-metabolomic analyses, bridging ecology and biochemistry (Peters et al. 2018), combined with laboratory approaches to properly improve our understanding of metabolic exchanges involved in actinorhizal symbioses.

\section{N storage, translocation, and regulation in the Frankia-Alnus symbiosis.}

Central role of Gln and Glu in $N$ metabolism. In bacteriaplant symbioses, $\mathrm{N}$ flux of metabolites is initiated by bacterial nitrogen fixation through the nitrogenase activity consisting in dinitrogen reduction to ammonium. This reaction is followed by ammonium assimilation through the GS/GOGAT cycle permitting the assimilation of ammonium to Gln and Glu before their conversion into other N compounds (Lurthy et al. 2018). Here, Gln and Glu showed very distinct patterns. Glu was one of the most abundant amino acids in nodule and root samples, with significantly higher abundances in nodules compared with roots, while Gln showed lower relative abundances in Alnus tissues, with a tendency to be more abundant in roots. Interestingly, both Gln and Glu, at the two concentrations tested, reduced nitrogen fixation and strongly stimulated respiration of Frankia cells when supplied in the culture medium, with a more sensitive response for Gln. Similar effects were observed, by the addition of ammonium to the medium, on Frankia nitrogen fixation and respiration (data not shown), suggesting their assimilation as nitrogen source, such as previously reported (Zhang and Benson 1992). In addition, it has been reported that during nitrogen fixation in pure culture, Frankia cells mainly accumulate Glu (Carro et al. 2015). The inhibiting effects of Gln and Glu on Frankia nitrogen fixation imply the need for the host plant to control their content in nodules in order to sustain its symbiont fixation activity. The concentrations of Gln or Glu seem not to reach those inhibiting concentrations at which the absolute concentration of $1 \mathrm{~g}$ of dried crushed nodule presented lower value (Supplementary Table S2). However, estimate the real concentration in the immediate proximity of bacteria is a challenging perspective, and promising approaches such as

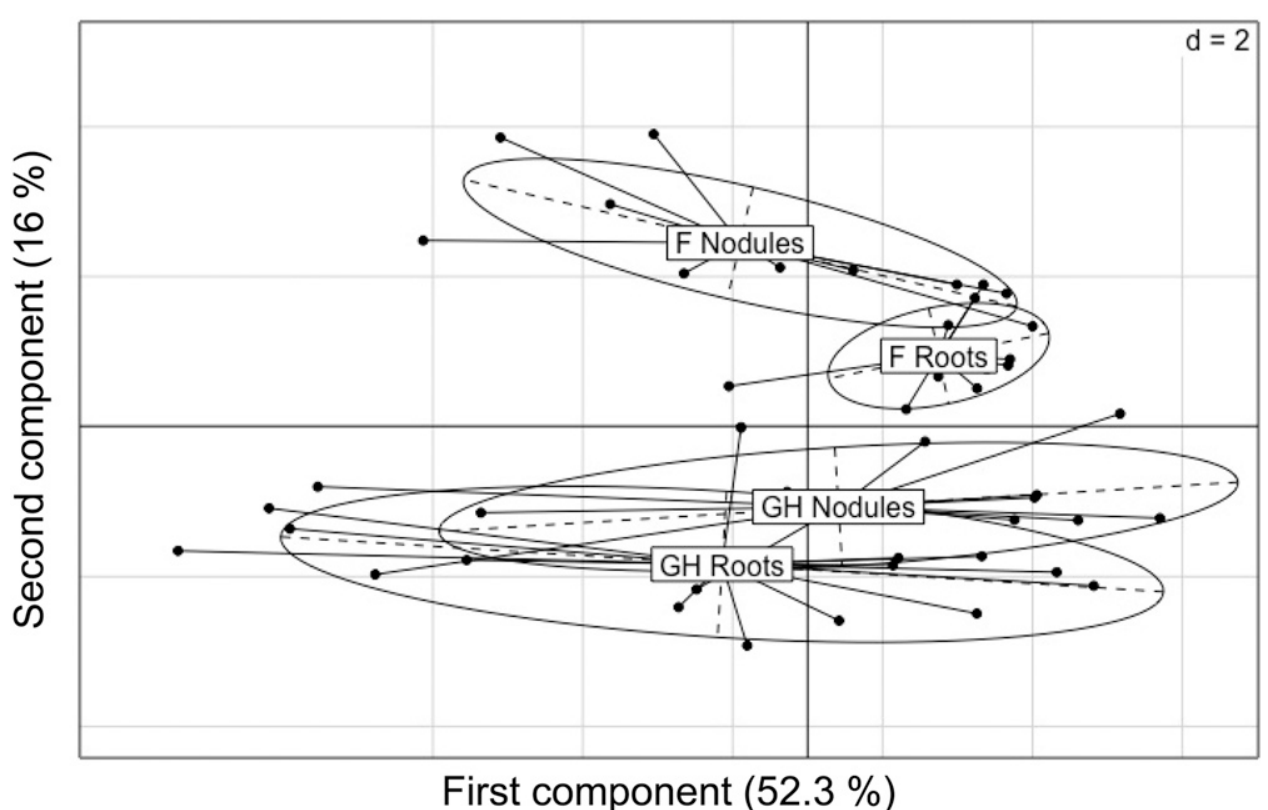

Fig. 2. Principal component analysis of nodules and root metabolic profiles for amino acids for each type of sample: field (F) or greenhouse (GH) for factors 1 (horizontal axis) and 2 (vertical axis) scored at 52.3 and $16 \%$, respectively, with a better separation of field nodule and root samples along the first axis. 
matrix-assisted laser desorption-imaging mass spectrometry (MALDI-IMS) could be developed to couple both amino acid localization and quantification of the different metabolites in the different plant compartments (Gemperline and Li 2015; Gemperline et al. 2015; Ye et al. 2013).

Primary nitrogen assimilation and $\mathrm{N}$-feedback control of the plant into its bacterial symbiont. Concerning the primary assimilation of ammonium from Frankia cells to plant cells, compilation of several results give us an incomplete picture. Indeed, very little GS activity $(<1 \%)$ and no protein has been detected in Frankia vesicle clusters extracted from Alnus nodules (Blom et al. 1981; Lundquist and Huss-Danell 1992), but, at the transcriptional level, a moderate upregulation of one GS (GSII) was observed, as compared with $\mathrm{N}$-depleted culture (Alloisio et al. 2010; Lurthy et al. 2018). Reciprocally, the GS/GOGAT enzymes of plant cells were upregulated (Guan et al. 1996) and specifically immunolocalized in infected cortical plant cells, but the authors could not exclude the possibility of GS presence in the endophyte (Guan et al. 1996; Hirel et al. 1982). In addition, no GOGAT enzyme activity was detected in Frankia vesicles (Schultz and Benson 1990) and no other bacterial genes involved in Arg pathways were modulated in A. glutinosa nodules (Berry et al. 2011). Taken together, the immediate products of nitrogen fixation exported from Frankia cells to the host could be ammonium and, also, Gln with or without another product at a lower level (Fig. 5). These hypotheses required further investigation through the specific detection of bacterial GS by immunolocalization coupled to electron microscopy and developing genetic tools.

In addition, the host plant would certainly provide a $\mathrm{N}$ compound to the symbiont as feedback, such as observed in leguminous symbiosis (Lodwig et al. 2003; Prell et al. 2009b; Rastogi and Watson 1991). Indeed, without N feedback, Frankia cells would be under nitrogen starvation, as observed in $\mathrm{N}$-fixing cultures through the overexpression of several proteins involved in ammonium scavenging, which is not seen in symbiosis (Lurthy et al. 2018). Because Glu is the amide acceptor of the GS reaction active in Frankia vesicles and is moderately active in Frankia cells in the Alnus nodule, we hypothesize that it could be provided to Frankia cells by the host plant as $\mathrm{N}$ feedback, but other $\mathrm{N}$ compounds could perform this function.

Concerning bacteria-plant cell exchanges, a possible flow of amino acids would occur through hyphae or vesicle walls (Fig. 5). It was recently reported that the host plant $A$. glutinosa secretes a plant defensin that specifically increases Frankia vesicle permeability, leading to the in-vitro efflux of $\mathrm{N}$ compounds and, particularly, Gln and Glu (Carro et al. 2015). Amino acid transporters such as Bra/Liv or Aap systems that play an important function in leguminous symbiosis (Lodwig
A

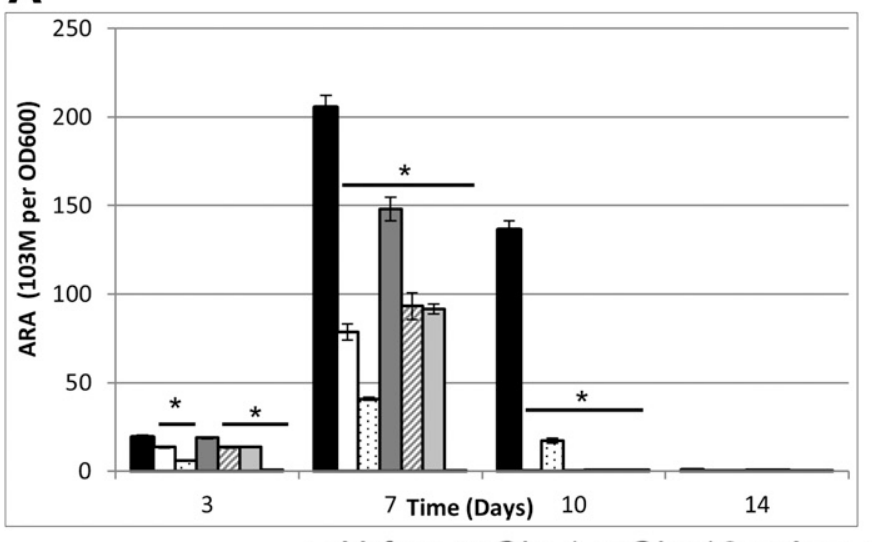

-N-free $\square$ Glu 1 घGlu $10 \square$ Asp

C

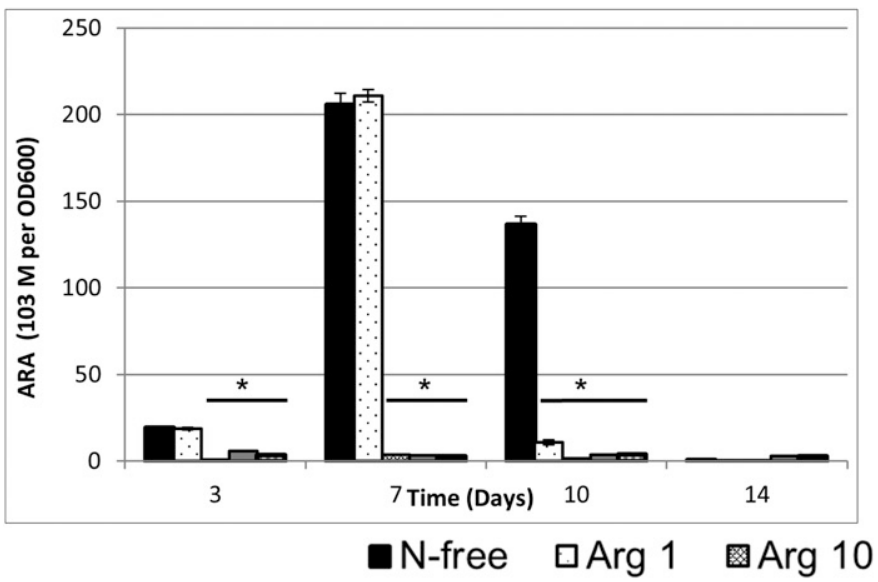

B

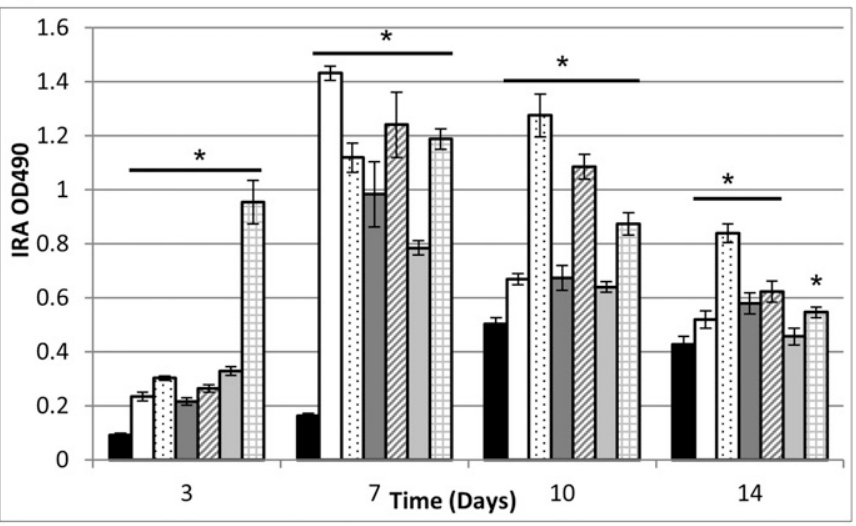

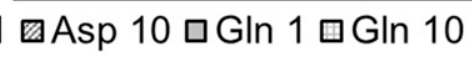

D

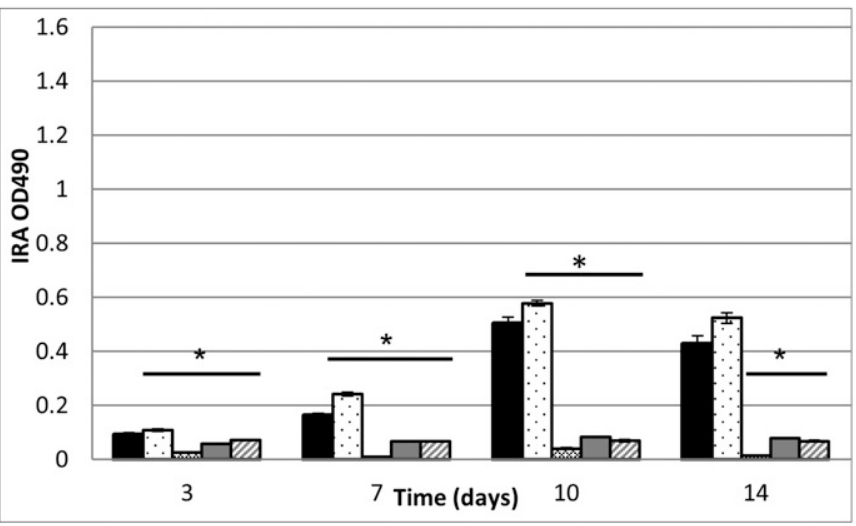

口His 1 虽 10

Fig. 3. A and B, Effects of glutamate (Glu), aspartate (Asp), glutamine (Gln) and $\mathbf{C}$ and $\mathbf{D}$, arginine (Arg) and histidine (His) on Frankia ACN14a nitrogen fixation (A, C) and respiration rates (B, D). Nitrogen activity was estimated through acetylene reduction activity, normalized to moles per liter at optical density of $600 \mathrm{~nm}$, as previously described (Carro et al. 2015a; Prin et al. 1990). Bacterial respiration was measured through iodonitrotetrazolium reduction activity at $490 \mathrm{~nm}$. Amino acids were tested at two different concentrations, 1 and $10 \mathrm{mM}$, under $\mathrm{N}$-fixing conditions ( $\mathrm{N}$-free media). Asterisks $(*)$ indicate significant difference observed compared with the control $(\mathrm{N}-$ free $)$ for the same time $(P$ value $<0.05)$. 
et al. 2003; Prell et al. 2009b; Rastogi and Watson 1991) could also be used in this model, although no bacterial or plant transporters have been described so far. Previous transcriptional analyses on $F$. alni ACN14a reported that several genes coding for putative amino acid transporters are moderately or totally inhibited in nodules compared with bacterial culture under $\mathrm{N}$ fixing conditions (e.g., FRAAL3079, FRAAL4701 [Supplementary Table S3]). In parallel, only two genes (FRAAL0792 and FRAAL3365) were slightly upregulated in nodules and could potentially be involved in the $\mathrm{N}$ compound flux but required more genetic investigation.

Cit and Asp involved in $N$ storage and translocation. Cit is the most abundant amino acid found in Alnus roots and nodules and has been considered as a main form of $\mathrm{N}$ storage (Berry et al. 2011; Valverde and Huss-Danell 2008). Here, the metabolic profiling of A. glutinosa associated roots and nodules presented the same pattern with a high mean relative abundance of cit of around 52\%. Interestingly, the Cit supplementation of Frankia ACN14a in $\mathrm{N}$-free medium significantly stimulated nitrogen fixation and bacterial respiration. This suggests that Cit is not taken up or assimilated as a nitrogen source by Frankia cells and, consequently, could be used as an N-storage compound by the plant without disturbing the N-fixation activity of the microbial symbiont. The moderate stimulation of Cit on bacterial respiration at a high concentration (Fig. 4B) could be explained by a possible assimilation as carbon nutrient. Cit could also serve as signaling amino acid, such as described for GABA and Glu (Ramesh et al. 2017; Toyota et al. 2018). Cit could also be a N-translocator compound from the nodule to the rest of the plant. Indeed, it has been recovered in alder sap as the main amino acid (Baker et al. 1997; Blom et al. 1981; Tonin et al. 1990) and it is usually described as being produced during the urea cycle, leading to Arg biosynthesis with ornithine as an intermediate (Fig. 5).

Similar to Cit, Asp could play a role in $\mathrm{N}$ translocation. It was found abundantly present in A. glutinosa tissues (around 6\%), with significantly higher concentrations in root samples (Fig. 1) and in sap metabolic profiles (Baker et al. 1997; Blom et al. 1981; Tonin et al. 1990). However, the in-vitro assay suggested that Asp is used as nitrogen nutrient by bacteria, similarly to Glu and Gln (Fig. 3). Controlling its concentration in nodules would, therefore, be crucial for the host plant to reduce Frankia nitrogen assimilation.
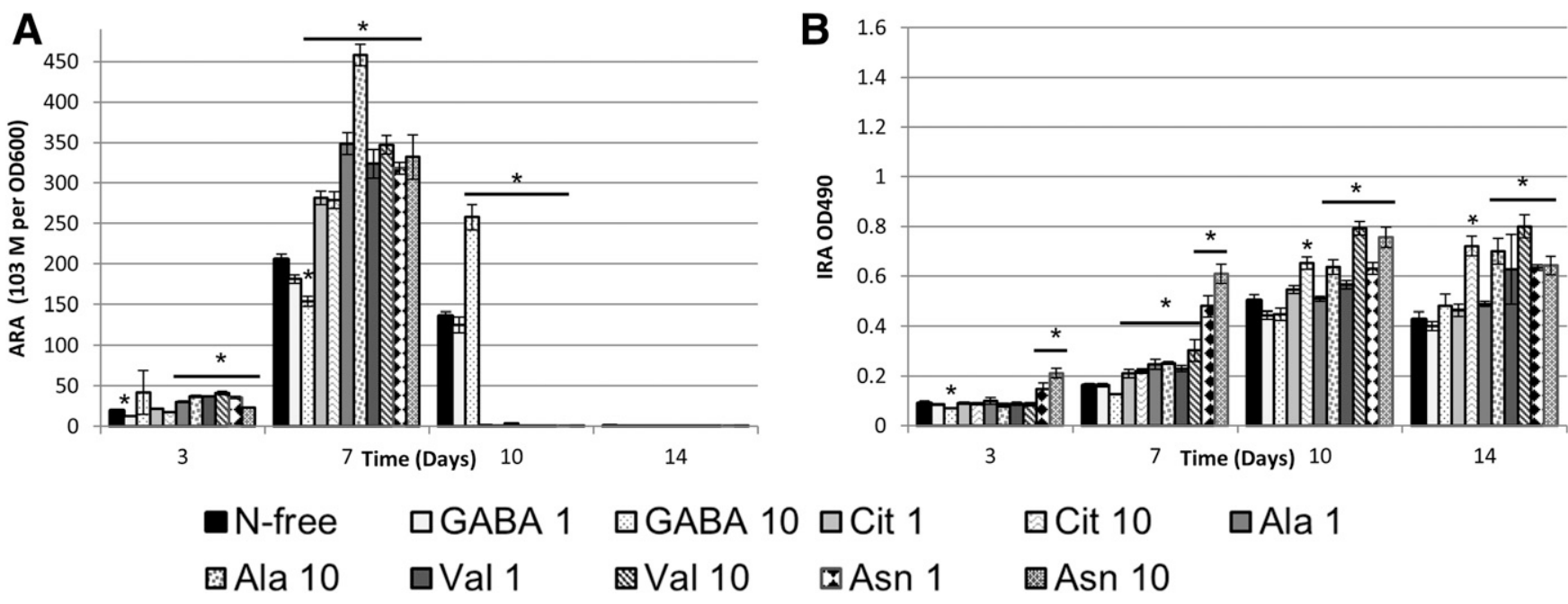

Fig. 4. A, Effects of $\gamma$-amino-n-butyric acid (GABA), citrulline (Cit), alanine (Ala), valine (Val), and asparagine (Asn) on Frankia ACN14a nitrogen fixation and $\mathbf{B}$, respiration rates. Amino acids were tested at two different concentrations, 1 and $10 \mathrm{mM}$, under $\mathrm{N}$-fixing conditions $(\mathrm{N}$-free media). Asterisks $(*)$ indicate significant difference observed compared with the control $(\mathrm{N}-$ free $)$ for the same time $(P$ value $<0.05)$.

504 / Molecular Plant-Microbe Interactions 
described (Pozzi et al. 2015) (Supplementary Table S1). Three different trees were randomly selected per site and, for each of them, a minimum of three nodules were collected with associated roots. All Frankia strains inhabiting field nodules used in this study had been previously genotyped (Pozzi et al. 2015). All collected material was stored at $-80^{\circ} \mathrm{C}$ before being freezedried (Christ Alpha 1-4 freeze dryer). In our experiments, a nodule sample is defined as a pool of three nodules collected on three different trees (one nodule per tree) coming from the same site, which represents a minimum of three nodule samples per site. Similar sampling was done for associated roots (at least three pooled root samples were also made per site). Each sample, whether nodule or associated root, was made from around $150 \mathrm{mg}$ of freeze-dried material, crushed to a very fine dry powder (Retsch TissueLyser II) and kept at $-20^{\circ} \mathrm{C}$ before extraction.

\section{Nodule and root samples from greenhouse:} plant growth and inoculation conditions.

A. glutinosa seeds sampled from a single tree (bank of the Rhone river in Lyon) were germinated on a sterilized mixture of soil/vermiculite $(1: 1, \mathrm{vol} / \mathrm{vol})$ for 5 weeks in a greenhouse (with a 16-h and 8-h nycthemeral regime at temperatures of 21 and $25^{\circ} \mathrm{C}$, respectively). Before bacterial inoculation, seedlings were transferred to Fåhraeus medium (Fahraeus 1957) with $5 \mathrm{mM} \mathrm{KNO}_{3}$ in plastic pots (four seedlings per pot) for 4 weeks, and then, on Fåhraeus medium without $\mathrm{KNO}_{3}$ for 2 weeks.

F. alni ACN14a (Normand and Lalonde 1982) and Frankia AgTrS (Bethencourt et al. 2019) were used for inoculation. The strain ACN14a is cultivable and was grown on $\mathrm{N}$ supplemented medium (FBM+) for 3 weeks followed by on an ammonium free medium (FBM-) for 2 weeks. FBM medium derived from modified BAP medium (Lurthy et al. 2018) by i) suppressing vitamins and ii) replacing iron citrate by iron chloride $\mathrm{FeCl}_{3}$ at a final concentration of $20 \mu \mathrm{M}$. The addition of $5 \mathrm{mM}$ of $\mathrm{NH}_{4} \mathrm{Cl}$ constituted the $\mathrm{FBM}+$ medium, as opposed to $\mathrm{N}$-fixing conditions with FBM-. Cultures were syringe-infiltrated through a $21 \mathrm{G}$ needle to fragment mycelium, and $4 \mathrm{ml}$ of the syringed culture was used per pot for inoculation (final volume in pot was around 1 liter). Strain AgTrS was chosen because it was previously observed as predominant in nodules from Tremblay, one of the four sampled sites (Pozzi et al. 2015). This strain has not been isolated and required using crushed nodules to inoculate the plant. For this, we applied the same procedure as Cotin-Galvan et al. (2016), using a suspension of crushed nodules freshly collected on the TrS site. Field nodules collected from this site were used for inoculating greenhouse seedlings and, 5 months after inoculation, DNA was extracted from young nodules. Housekeeping genes ( $d n a A, f t s Z$, and $p g k)$ amplification and sequencing were performed, as previously described (Pozzi et al. 2015), to confirm the identity of the Frankia genotype in nodules (data not shown). Roots and nodules were harvested from nodulated plants (six to eight plants per condition) and were stored at $-80^{\circ} \mathrm{C}$ before amino acid analyses.

\section{Amino acid extraction from nodule and root samples.}

A total of 24 nodule and root samples from the field and 28 samples from the greenhouse were extracted. Before extraction, $0.1 \mathrm{nmol}$ of norvaline per milligram was added as an internal standard in each sample. Two successive extractions were performed, in $1 \mathrm{ml}$ of ethanol and water $(60: 40 \mathrm{vol} / \mathrm{vol})$ for $100 \mathrm{mg}$ of sample, by vortexing (Heidolph Top-mix 94323) and sonicating (Bransonic Ultrasonic cleaner 2510E-DTH) for $15 \mathrm{~min}$, followed by $10 \mathrm{~min}$ of centrifugation at $13,000 \times g$. The resulting supernatants were kept as the first extracts (E1 and E2). The remaining pellet was used for the third extraction, performed in $100 \%$ water ( $1 \mathrm{ml}$ to $100 \mathrm{mg}$ sample) followed by dissolving, as before, to get the third extract (E3). For each sample, all three supernatants were pooled and dried (CentriVap concentrator $\mathrm{LABCONCO})$. They were stored at $-20^{\circ} \mathrm{C}$, prior to chromatographic analysis.

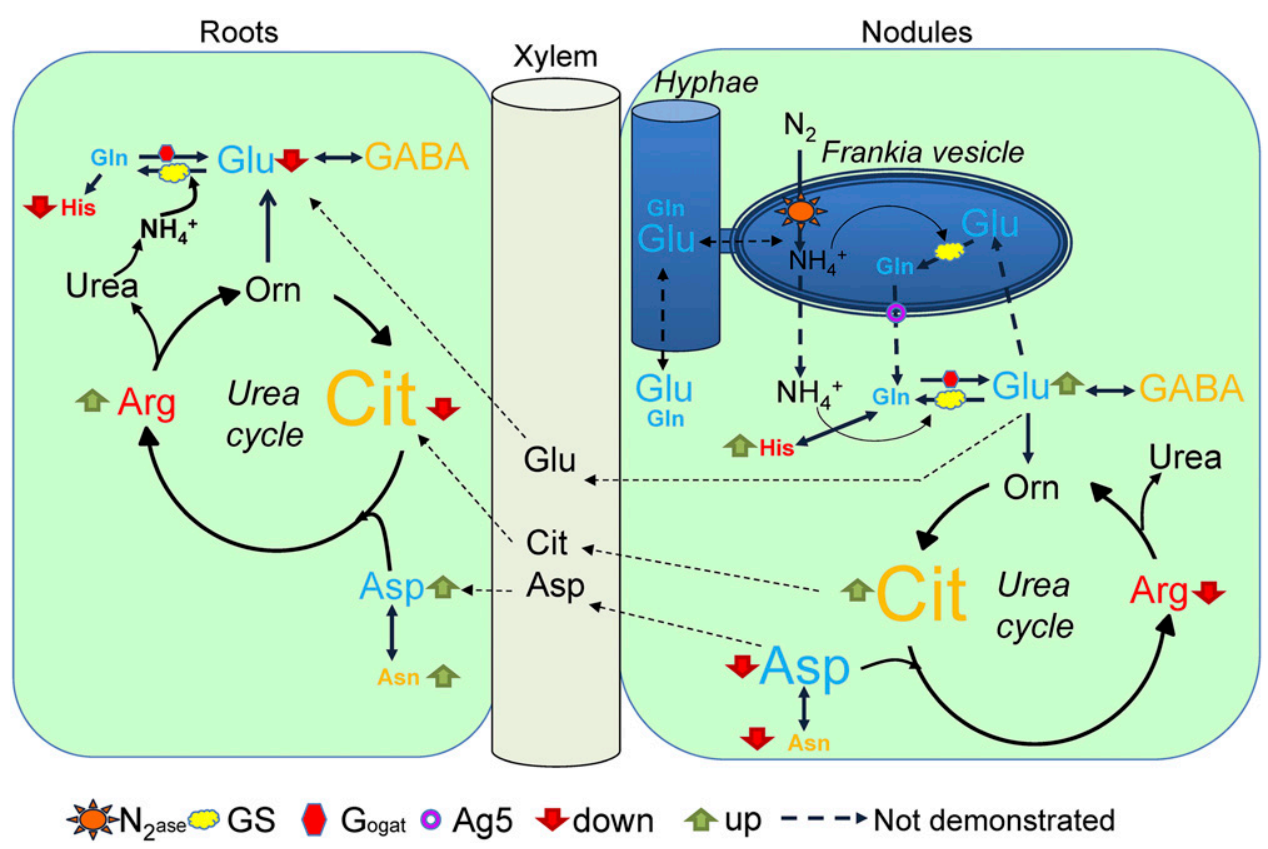

Fig. 5. Suggested pathways for the assimilation of ammonium in Alnus-Frankia root nodules. The font size of each amino acid illustrates their abundance in Alnus tissues. Their effect on Frankia in-vitro assays is indicated by colored font with red as toxic, blue as nitrogen source, and yellow as neutral effect or putative carbon source. Full and thin dotted lines represent pathway reactions and nodule-xylem-root metabolic exchanges, respectively. Thick dotted lines represent hypothetical pathway reactions. Several proteins are illustrated: Cit $=$ citrulline, Arg $=$ arginine, Asp $=$ aspartate, Gln $=$ glutamine, $\mathrm{N}_{2}$ ase $=$ nitrogenase, GS = Gln synthetase, GOGAT = Gln oxoglutarate aminotransferase or Glu synthase, and Ag5 = the plant defensin. The relative abundance of amino acids that decreased (down) or increased (up) in each tissue (from either greenhouse or field samples or both) was represented by a red or a green arrow, respectively. 


\section{Chromatographic analysis of amino acid contents in nodule and root extracts.}

For amino acid chromatographic analyses, extracts were solubilized in $100 \%$ water in order to get a norvalin concentration of $50 \mu \mathrm{M}$. Chromatographic analysis was performed by high-pressure liquid chromatography (HPLC) (Agilent 1100; Agilent Technologies) with a guard cartridge and a C18 reverse phase column (Zorbax Eclipse-AAA $3.5 \mathrm{~mm}, 150 \times 4.6 \mathrm{~mm}$; Agilent Technologies), according to the procedure specifically developed for this system (Henderson et al. 2000). These conditions do not allow detection and quantification of cysteine and methionine, and hence, only 20 proteogenic and nonproteogenic amino acids were quantified: Ala, Arg, Asn, Asp, Cit, GABA, Gln, Glu, Gly, His, Ile, Leu, Lys, Phe, Pro, Ser, Thr, Trp, Tyr, and Val. For this quantification, norvaline was used as the internal standard and the response factor of each amino acid was determined using a $250 \mathrm{pmol} \mathrm{ml}^{-1}$ standard mix of each amino acid. The software used was the ChemStation for LC 3D systems B01.03 (Agilent Technologies). For Arg and Cit, detection was done using UV at $338 \mathrm{~nm}$. For all amino acids, absolute concentrations in microMorgans in the extracts were calculated from UV or fluorescence chromatogram, using Cabs $=($ CNor $\times$ Aabs $) /($ Anor $\times \mathrm{CR})$, where Cabs is absolute concentrations, CNor is the concentration of norvalin in microMorgans, Aabs the absolute area for amino acids, ANor the absolute area for norvaline, and CR the ratio between areas of amino acids and norvalin. The presence of the internal standard norvalin allowed the comparison of absolute concentrations of amino acids within a sample and for each amino acid between different samples. Relative concentrations were expressed as percentages, for each amino acid by calculating Crel $(\%)=$ $((\mathrm{Cabs}) /(\mathrm{Ctot})) \times 100$, where Cabs is expressed in microMorgans and Ctot is the sum of all the Cabs for one sample (in microMorgan). Concentrations in microMorgans were converted to micromoles per gram of dried material, by calculating $\mathrm{Cabs}_{(\mu \mathrm{mol} / \mathrm{gram})}=(\mathrm{Cabs} \times \mathrm{V}) / \mathrm{m}_{\text {sample }}$, where $\mathrm{V}$ is the volume of water used for each sample to get the concentration of $50 \mu \mathrm{M}$ of Norvalin and $\mathrm{m}_{\text {sample }}$ is the quantity of dried material of nodules or roots.

PCAs were made from these matrices in order to visualize the effect of sample origin (field versus greenhouse) and plant organ (nodule versus root) on amino acid profiles. The effect of plant organ on amino acid relative abundances was also statistically tested, in order to highlight significantly different metabolites between nodules and roots. Statistical analyses were performed using $\mathrm{R}$ software version 2.15.3. Since data were not normally distributed as determined by Shapiro and Bartlett tests (Bartlett and Fowler 1937; Shapiro and Wilk 1965), the Kruskal Wallis statistical test (Kruskal and Wallis 1952) was used.

\section{Frankia ACN14a strain growth conditions and in-vitro tests of amino acids.}

F. alni ACN14a was grown in FBM medium. Addition of $5 \mathrm{mM}$ of $\mathrm{NH}_{4} \mathrm{Cl}$ constituted $\mathrm{FBM}+$ as opposed to FBMwithout ammonium. Amino acids were solubilized in FBMmedia at a final concentration of $10 \mathrm{mM}$, adjusted at $\mathrm{pH} 6.3$, and were diluted to $1 \mathrm{mM}$ in the same medium. Two-week-old cultures with two induction steps in FBM- medium were made before in-vitro tests. Cultures were syringed through a series of 21G, 23G, 25G, and 26G needles. This Frankia ACN14a inoculum was added to FBM- at a final optical density of 0.1 . Five biological replicates were performed for each amino acid and positive and negative controls were also included by testing without amino acid or without Frankia inoculum. After 3, 7, 10, and 14 days of growth, the effects of amino acids were tested on growth (optical density at $600 \mathrm{~nm}$ ), bacterial respiration (through IRA) and nitrogen fixation (through ARA) as described previously (Carro et al. 2016a; Prin et al. 1990). Gln was previously described as playing an important role to control bacterial nitrogen fixation (Schumacher et al. 2013; Zhang and Benson 1992) and was used here to cause ARA inhibition. Since data were not normally distributed, the Kruskal Wallis test was used ( $\mathrm{R}$ software version 3.4.2).

\section{ACKNOWLEDGMENTS}

Thanks are expressed to "FR Bio-Environment Santé" for use of the greenhouse platform and to AME ("Activités Microbiennes dans l'Environnement") platform (UMR5557) for use of gas chromatography for ARA analysis. We thank P. Pujic, L. Carro, and M. J. Tulumello for technical advice.

\section{LITERATURE CITED}

Alloisio, N., Queiroux, C., Fournier, P., Pujic, P., Normand, P., Vallenet, D., Médigue, C., Yamaura, M., Kakoi, K., and Kucho, K. 2010. The Frankia alni symbiotic transcriptome. Mol. Plant-Microbe Interact. 23:593-607.

Baker, A., Hill, G. F., and Parsons, R. 1997. Evidence for N feedback regulation of $\mathrm{N}_{2}$ fixation in Alnus glutinosa L. J. Exp. Bot. 48: 67-73.

Bartlett, M. S., and Fowler, R. H. 1937. Properties of sufficiency and statistical tests. Proc. R. Soc. Lond. A Math. Phys. Sci. 160:268-282.

Berry, A. M., Harriott, O. T., Moreau, R. A., Osman, S. F., Benson, D. R., and Jones, A. D. 1993. Hopanoid lipids compose the Frankia vesicle envelope, presumptive barrier of oxygen diffusion to nitrogenase. Proc. Natl. Acad. Sci. U.S.A. 90:6091-6094.

Berry, A. M., Mendoza-Herrera, A., Guo, Y.-Y., Hayashi, J., Persson, T., Barabote, R., Demchenko, K., Zhang, S., and Pawlowski, K. 2011. New perspectives on nodule nitrogen assimilation in actinorhizal symbioses. Funct. Plant Biol. 38:645-652.

Berry, A. M., Murphy, T. M., Okubara, P. A., Jacobsen, K. R., Swensen, S. M., and Pawlowski, K. 2004. Novel expression pattern of cytosolic Gln synthetase in nitrogen-fixing root nodules of the actinorhizal host, Datisca glomerata. Plant Physiol. 135:1849-1862.

Bethencourt, L., Vautrin, F., Taib, N., Dubost, A., Castro-Garcia, L., Imbaud, O., Abrouk, D., Fournier, P., Briolay, J., Nguyen, A., Normand P., Fernandez, M. P., Brochier-Armanet, C., and Herrera-Belaroussi, A. 2019. Draft genome sequences for three unisolated Alnus-infective Frankia Sp+ strains, AgTrS, AiOr and AvVan, the first sequenced Frankia strains able to sporulate in-planta. J Genomics 7:50-55.

Blom, J., Roelofsen, W., and Akkermans, A. D. L. 1981. Assimilation of nitrogen in root nodules of alder (Alnus glutinosa). New Phytol 89: 321-326.

Brooks, J. M., and Benson, D. R. J. S. 2016. Comparative metabolomics of root nodules infected with Frankia sp. strains and uninfected roots from Alnus glutinosa and Casuarina cunninghamiana reflects physiological integration. Symbiosis 70:87-96.

Carro, L., Persson, T., Pujic, P., Alloisio, N., Fournier, P., Boubakri, H., Pawlowski, K., and Normand, P. 2016a. Organic acids metabolism in Frankia alni. Symbiosis 70:37-48.

Carro, L., Pujic, P., Alloisio, N., Fournier, P., Boubakri, H., Hay, A. E., Poly, F., François, P., Hocher, V., Mergaert, P., Balmand, S., Rey, M., Heddi, A., and Normand, P. 2015. Alnus peptides modify membrane porosity and induce the release of nitrogen-rich metabolites from nitrogen-fixing Frankia. ISME J. 9:1723-1733.

Carro, L., Pujic, P., Alloisio, N., Fournier, P., Boubakri, H., Poly, F., Rey, M., Heddi, A., and Normand, P. 2016b. Physiological effects of major up-regulated Alnus glutinosa peptides on Frankia sp. ACN14a. Microbiology 162:1173-1184.

Carro, L., Pujic, P., Trujillo, M. E., and Normand, P. 2013. Micromonospora is a normal occupant of actinorhizal nodules. J. Biosci. 38:685-693.

Cotin-Galvan, L., Pozzi, A.C., Schwob, G., Fournier, P., Fernandez, M. P., and Herrera-Belaroussi, A. 2016. In-planta sporulation capacity enhances infectivity and rhizospheric competitiveness of Frankia strains. Microbes Environ. 31:11-18.

Dos Santos, P. C., Fang, Z., Mason, S. W., Setubal, J. C., and Dixon, R. 2012. Distribution of nitrogen fixation and nitrogenase-like sequences amongst microbial genomes. BMC Genomics 13:162.

Fahraeus, G. 1957. The infection of clover root hairs by nodule bacteria studied by a simple glass slide technique. J. Gen. Microbiol. 16 374-381. 
Gardner, I. C., and Leaf, G. 1960. Translocation of citrulline in Alnus glutinosa. Plant Physiol. 35:948-950.

Gemperline, E., Jayaraman, D., Maeda, J., Ané, J. M., and Li, L. 2015 Multifaceted investigation of metabolites during nitrogen fixation in Medicago via high resolution MALDI-MS imaging and ESI-MS. J. Am. Soc. Mass Spectrom. 26:149-158.

Gemperline, E., and Li, L. 2015. MALDI-MS-assisted molecular imaging of metabolites in legume plants. Methods Mol. Biol. 1203:29-40.

Ghodhbane-Gtari, F., Essoussi, I., Chattaoui, M., Chouaia, B., Jaouani, A., Daffonchio, D., Boudabous, A., and Gtari, M. 2010. Isolation and characterization of non-Frankia actinobacteria from root nodules of Alnus glutinosa, Casuarina glauca and Elaeagnus angustifolia. Symbiosis 50:51-57.

Gifford, I., Vance, S., Nguyen, G., and Berry, A. M. 2019. A Stable genetic transformation system and implications of the type IV restriction system in the nitrogen-fixing plant endosymbiont Frankia alni ACN14a. Front. Microbiol. 10:2230.

Griesmann, M., Chang, Y., Liu, X., Song, Y., Haberer, G., Crook, M. B., Billault-Penneteau, B., Lauressergues, D., Keller, J., Imanishi, L., Roswanjaya, Y. P., Kohlen, W., Pujic, P., Battenberg, K., Alloisio, N., Liang, Y., Hilhorst, H., Salgado, M. G., Hocher, V., Gherbi, H., Svistoonoff, S., Doyle, J. J., He, S., Xu, Y., Xu, S., Qu, J., Gao, Q., Fang, X., Fu, Y., Normand, P., Berry, A. M., Wall, L. G., Ané, J. M., Pawlowski, K., Xu, X., Yang, H., Spannagl, M., Mayer, K. F. X., Wong, G. K., Parniske, M., Delaux, P. M., and Cheng, S. 2018. Phylogenomics reveals multiple losses of nitrogen-fixing root nodule symbiosis. Science 361:eaat1743.

Guan, C., Ribeiro, A., Akkermans, A. D., Jing, Y., van Kammen, A., Bisseling, T., and Pawlowski, K. 1996. Nitrogen metabolism in actinorhizal nodules of Alnus glutinosa: Expression of glutamine synthetase and acetylornithine transaminase. Plant Mol. Biol. 32:1177-1184.

Hay, A. E., Boubakri, H., Buonomo, A., Rey, M., Meiffren, G., CotinGalvan, L., Comte, G., and Herrera-Belaroussi, A. 2017. Control of endophytic Frankia sporulation by Alnus nodule metabolites. Mol. PlantMicrobe Interact. 30:205-214.

Henderson, J., Bicker, R., Bidlingmeyer, B., and Woodward, C. 2000. Rapid, accurate, sensitive, and reproducible HPLC analysis of aminoacids: Amino acid analysis using Zorbax Eclipse-AAA columns and the Agilent 1100 HPLC. Agilent Technologies, Santa Clara, CA, U.S.A.

Hirel, B., Perrot-Rechenmann, C., Maudimas, B., and Gadal, P. 1982 Glutamine synthetase in alder (Alnus glutinosa) root nodules: Purification, properties and cytoimmunochemical localization. Physiol. Plant 55: 197-203.

Hocher, V., Alloisio, N., Bogusz, D., and Normand, P. 2011. Early signaling in actinorhizal symbioses. Plant Signal. Behav. 6:1377-1379.

Kacprzyk, L., Rydengård, V., Mörgelin, M., Davoudi, M., Pasupuleti, M., Malmsten, M., and Schmidtchen, A. 2007. Antimicrobial activity of histidine-rich peptides is dependent on acidic conditions. Biochim. Biophys. Acta 1768:2667-2680.

Kruskal, W. H., and Wallis, W. A. 1952. Use of ranks in one-criterion variance analysis. J. Am. Stat. Assoc. 47:583-621.

Kucho, K., Kakoi, K., Yamaura, M., Higashi, S., Uchiumi, T., and Abe, M. 2009. Transient transformation of frankia by fusion marker genes in liquid culture. Microbes Environ. 24:231-240.

Leaf, G., Bond, G., and Gardner, I. C. 1958. Observations on the composition and metabolism of the nitrogen-fixing root nodules of Alnus. J. Exp. Bot. 9:320-331.

Lodwig, E. M., Hosie, A. H., Bourdès, A., Findlay, K., Allaway, D., Karunakaran, R., Downie, J. A., and Poole, P. S. 2003. Amino-acid cycling drives nitrogen fixation in the legume-Rhizobium symbiosis. Nature 422:722-726.

Lundberg, P., and Lundquist, P. O. 2004. Primary metabolism in $\mathrm{N}_{2}$-fixing Alnus incana-Frankia symbiotic root nodules studied with $15 \mathrm{~N}$ and $31 \mathrm{P}$ nuclear magnetic resonance spectroscopy. Planta 219:661-672.

Lundquist, P.-O., and Huss-Danell, K. 1992. Immunological studies of glutamine synthetase in Frankia-Alnus incana symbioses. FEMS Microbiol. Lett. 91:141-146.

Lurthy, T., Alloisio, N., Fournier, P., Anchisi, S., Ponsero, A., Normand, P., Pujic, P., and Boubakri, H. 2018. Molecular response to nitrogen starvation by Frankia alni ACN14a revealed by transcriptomics and functional analysis with a fosmid library in Escherichia coli. Res. Microbiol. 169:90-100

Martin, F. M., Uroz, S., and Barker, D. G. 2017. Ancestral alliances: Plant mutualistic symbioses with fungi and bacteria. Science 356:eaad4501.

Molero, G., Aranjuelo, I., Teixidor, P., Araus, J. L., and Nogués, S. 2011. Measurement of $13 \mathrm{C}$ and $15 \mathrm{~N}$ isotope labeling by gas chromatography/combustion/isotope ratio mass spectrometry to study amino acid fluxes in a plant-microbe symbiotic association. Rapid Commun. Mass Spectrom. 25:599-607
Normand, P., and Lalonde, M. 1982. Evaluation of Frankia strains isolated from provenances of two Alnus species. Can. J. Microbiol. 28:1133-1142.

Pawlowski, K., Twigg, P., Dobritsa, S., Guan, C., and Mullin, B. C. 1997. A nodule-specific gene family from Alnus glutinosa encodes glycine- and histidine-rich proteins expressed in the early stages of actinorhizal nodule development. Mol. Plant-Microbe Interact. 10:656-664.

Persson, T., Van Nguyen, T., Alloisio, N., Pujic, P., Berry, A. M., Normand, P., and Pawlowski, K. 2016. The N-metabolites of roots and actinorhizal nodules from Alnus glutinosa and Datisca glomerata: Can D. glomerata change N-transport forms when nodulated? Symbiosis 70:149-157.

Pesce, C., Oshone, R., Hurst, S. G., Kleiner, V. A., and Tisa, L. S. 2019. Stable transformation of the actinobacteria Frankia. Appl. Environ. Microb. 85:e00957-19.

Peters, K., Worrich, A., Weinhold, A., Alka, O., Balcke, G., Birkemeyer, C., Bruelheide, H., Calf, O. W., Dietz, S., Dührkop, K., Gaquerel, E., Heinig, U., Kücklich, M., Macel, M., Müller, C., Poeschl, Y., Pohnert, G., Ristok, C., Rodríguez, V. M., Ruttkies, C., Schuman, M., Schweiger, R., Shahaf, N., Steinbeck, C., Tortosa, M., Treutler, H., Ueberschaar, N., Velasco, P., Weiß, B. M., Widdig, A., Neumann, S., and Dam, N. M. V. 2018. Current challenges in plant eco-metabolomics. Int. J. Mol. Sci. 19:1385.

Pozzi, A. C., Bautista-Guerrero, H. H., Nouioui, I., Cotin-Galvan, L., Pepin, R., Fournier, P., Menu, F., Fernandez, M. P., and Herrera-Belaroussi, A 2015. In-planta sporulation phenotype: A major life history trait to understand the evolution of Alnus-infective Frankia strains. Environ. Microbiol. 17:3125-3138.

Prell, J., Bourdès, A., Karunakaran, R., Lopez-Gomez, M., and Poole, P. 2009a. Pathway of $\gamma$-aminobutyrate metabolism in Rhizobium leguminosarum 3841 and its role in symbiosis. J. Bacteriol. 191:2177-2186.

Prell, J., and Poole, P. 2006. Metabolic changes of rhizobia in legume nodules. Trends Microbiol. 14:161-168.

Prell, J., White, J. P., Bourdes, A., Bunnewell, S., Bongaerts, R. J., and Poole, P. S. 2009b. Legumes regulate Rhizobium bacteroid development and persistence by the supply of branched-chain amino acids. Proc. Natl. Acad. Sci. U.S.A. 106:12477-12482.

Prin, Y., Neyra, M., and Diem, H. G. 1990. Estimation of Frankia growth using Bradford protein and INT reduction activity estimations: Application to inoculum standardization. FEMS Microbiol. Lett. 69:91-95.

Ramesh, S. A., Tyerman, S. D., Gilliham, M., and Xu, B. 2017. $\gamma$-Aminobutyric acid (GABA) signalling in plants. Cell. Mol. Life Sci. 74:1577-1603.

Rastogi, V. K., and Watson, R. J. 1991. Aspartate aminotransferase activity is required for aspartate catabolism and symbiotic nitrogen fixation in Rhizobium meliloti. J. Bacteriol. 173:2879-2887.

Schubert, K. R., and Coker, G. T. 1981. Ammonia assimilation in Alnus glutinosa and Glycine max: Short-term studies using $\left[{ }^{13} \mathrm{~N}\right] \mathrm{ammonium}$ Plant Physiol. 67:662-665.

Schultz, N. A., and Benson, D. R. 1990. Enzymes of ammonia assimilation in hyphae and vesicles of Frankia sp. strain CpI1. J. Bacteriol. 172: 1380-1384.

Schumacher, J., Behrends, V., Pan, Z., Brown, D. R., Heydenreich, F., Lewis, M. R., Bennett, M. H., Razzaghi, B., Komorowski, M., Barahona, M., Stumpf, M. P. H., Wigneshweraraj, S., Bundy, J. G., and Buck, M. 2013. Nitrogen and carbon status are integrated at the transcriptional level by the nitrogen regulator NtrC in vivo. MBio 4:e00881-13.

Schwob, G., Roy, M., Manzi, S., Pommier, T., and Fernandez, M. P. 2017. Green alder (Alnus viridis) encroachment shapes microbial communities in subalpine soils and impacts its bacterial or fungal symbionts differently. Environ. Microbiol. 19:3235-3250.

Schwob, G., Roy, M., Pozzi, A. C., Herrera-Belaroussi, A., and Fernandez, M. P. 2018. In planta sporulation of Frankia spp. as a determinant of alder-symbiont interactions. Appl. Environ. Microbiol. 84:e01737-18.

Sepahi, M., Jalal, R., and Mashreghi, M. 2017. Antibacterial activity of poly-1-arginine under different conditions. Iran. J. Microbiol. 9:103-111.

Shapiro, S. S., and Wilk, M. B. 1965. An analysis of variance test for normality (complete samples). Biometrika 52:591-611.

Tonin, G. S., Wheeler, C. T., and Crozier, A. 1990. Effect of nitrogen nutrition on amino acid composition of xylem sap and stem wood in Alnus glutinosa. Physiol. Plant. 79:506-511.

Toyota, M., Spencer, D., Sawai-Toyota, S., Jiaqi, W., Zhang, T., Koo, A. J., Howe, G. A., and Gilroy, S. 2018. Glutamate triggers long-distance, calcium-based plant defense signaling. Science 361:1112-1115.

Valverde, C., and Huss-Danell, K. 2008. Carbon and nitrogen metabolism in actinorhizal nodules. Pages 167-198 in: Nitrogen-fixing Actinorhizal Symbioses. K. Pawlowski, and W. E. Newton, eds. Springer Netherlands, Dordrecht.

Virtanen, A. I., and Miettinen, J. K. 1952. Free amino-acids in the leaves, roots and root nodules of the alder (Alnus). Nature 170:283-284. 
Watanabe, D., Kikushima, R., Aitoku, M., Nishimura, A., Ohtsu, I., Nasuno, R., and Takagi, H. 2014. Exogenous addition of histidine reduces copper availability in the yeast Saccharomyces cerevisiae. Microbial. Cell 1:241-246.

Wheeler, C. T., and Bond, G. 1970. The amino acids of non-legume root nodules. Phytochemistry 9:705-708.

Ye, H., Gemperline, E., Venkateshwaran, M., Chen, R., Delaux, P. M., Howes-Podoll, M., Ané, J. M., and Li, L. 2013. MALDI mass spectrometry-assisted molecular imaging of metabolites during nitrogen fixation in the Medicago truncatula-Sinorhizobium meliloti symbiosis. Plant J. 75:130-145.

Zehr, J. P., Jenkins, B. D., Short, S. M., and Steward, G. F. 2003. Nitrogenase gene diversity and microbial community structure: A crosssystem comparison. Environ. Microbiol. 5:539-554.

Zhang, X., and Benson, D. R. 1992. Utilization of amino acids by Frankia sp. strain CpI1. Arch. Microbiol. 158:256-261. 